hinreichend angeregt. Eine Lösung dieses Problems bestïnde darin, Emissionsrechte nicht für alle Zeiten zu verkaufen, sondern nur für einen bestimmten Zeitraum zu verpachten. Diese Vorgehensweise ermöglicht den Industrienationen einen langsamen und berechenbaren Ausstieg aus ihrer derzeitigen Technik und senkt so die Kosten des Klimaschutzes.

Das oben angesprochene Problem des 'hot air trading' ist weniger ein Problem des Handels als vielmehr eines der Wahl des Basisjahrs. Natürlich könnte man versuchen, auf Folgekonferenzen realistischere Basisszenarien mit strengeren Vorgaben $\mathrm{zu}$ verhandeln. Ein Erfolg erscheint jedoch zweifelhaft, da weder der derzeitige Hauptemittent USA noch die potentiellen Verkäufer von Emissionsrechten ein Interesse an Nachverhandlungen haben. Es wäre jedoch falsch, durch Mindestvermeidungsklauseln das Verhandlungsergebnis indirekt nachzubessern, da dies die Entwicklung eines Instruments behindert, dessen effizientes Funktionieren die Vereinbarung zukünftiger Protokolle erleichtert.

Auch wenn die Reduktionsziele des Kioto-Protokolls aus wissenschaftlicher Sicht enttäuschend gering sind, können vom Handel mit Emissionsrechten wichtige Impulse für eine Erweiterung der Teilnehmerländer und somit einen effektiveren Klimaschutz ausgehen. Selbst ein sich langsam entwickelnder Handel gibt das Signal, daß die Partizipation am Klimaschutz mit einem Zufluß an Finanzmitteln verbunden sein kann. Anreize zum 'free rider'-Verhalten werden so reduziert.

\section{Anmerkungen}

(1) WBGU: Ziele für den Klimaschutz 1997. Stellungnahme zur dritten Vertragsstaatenkonferenz der Klimarahmenkonvention in Kyoto, Wissenschaftlicher Beirat der Bundesregierung Globale Umweltverönderungen 1997.

(2) Vgl. Wiener, J.B.: Designing Markets for International Greenhouse Gas Control, RFF Climate Issues Brief \#6, Internet Edition, 1997; sowie UNCTAD: Legal Issues Presented by a Pilot International Greenhouse Gas Trading System, UNCTAD/GDS/GFSB/Misc. 1, 1997.

(3) Mullins, F., R.Baron: International GHG Emission Trading, Annex 1 Expert Group on the UN FCCC, Working Paper 9, 1997.

\section{Der Autor}

Or. Wilhelm Althammer ist Prolessor für Makroökonomie an der Handelshochschule Leipzig.

Kontokt: Handelshochschule Leipzig, Jahnallee 59, 04109 leipzig. Tel. 0341/9851-656, Fax: -659.

E-mail: althamm@macroec.hhl.de

Nicht-Regierungs-Organisationen als neue Akteure transnationaler (Umwelt-)Politik

\title{
Substitut des Staatsvolkes?
}

\author{
In der globalisierten Wirtschaft erodieren die klaren Konturen von Staatsge- \\ bief, Staatsvolk und Stautsmacht. Angesichts dieser Entgrenzung gewinnt das \\ Projekt von Global Governance seine Bedeutung. Nicht-Regierungs-Organisa- \\ tionen übernehmen darin eine wichtige Funktion.
}

B Von Elmar Altvater, Achim Brunnengräber und Heike Walk is vor nicht allzu langer Zeit bildeten Nationalstaaten und internationale Institutionen (und Organisationen) den geschlossenen Club der globalen Akteure. Die Welt war in erster Linie Staatenwelt, die sich eine internationale Ordnung gab und „Regime“ zur Regulierung von Handels- und Finanzfragen, zur Bewältigung von Umweltproblemen und zur Abwicklung der Entwicklungshilfe bildeten.

Infolge der Globalisierung aber haben diese traditionellen Akteure eines internationalen politischen Systems gegenüber „den Märkten“ an Einfluß verloren. Die Lösung der vielfältigen Probleme auf der internationalen Ebene wird daher nicht von den nationalstaatlichen Regierungen, aber auch nicht vom freien Spiel der Marktkräfte erwartet. Sie scheint nur noch möglich $\mathrm{zu}$ sein, wenn die Ressourcen der sogenannten ,Zivilgesellschaften“ aller Kontinente mobilisiert werden. Diese neue Konstellation von Akteuren hat auch die ,Commission on Global Governance" vor Augen, wenn sie in ihrem Bericht von 1994 feststellt, daß ,die großen Herausforderungen des folgenden Jahrzehnts nur durch multilateral abgestimmte Vorgehensweisen zu bewältigen seien“ (1) (S.395). Der Bericht der Kommission unterbreitet Handlungsempfehlungen und eröffnet einen Interpretationsspielraum, der bereits dem „ContainerBegriff“ Sustainable Development des Brundtland-Berichtes zur Karriere verhalf: es paßt sehr viel hinein (vgl. Stichwort: Global Governance in diesem Heft). Das Konzept der Global Governance läßt sich allerdings mit demjenigen der zukunftsorientierten Entwicklung systematisch verknüpfen: Die themen- und problemorientierte und die ordnungs- und akteursspezifische Perspektive werden zusammengebracht. So wird die symbolische Integrationskraft für unterschiedliche Handlungs- und Konfliktfelder erzeugt, die notwendig ist, um die
Schnittstelle zwischen der globalen Politik der Nachhaltigkeit und den Akteuren, die für diese Politik verantwortlich zeichnen sollen, zum Funktionieren zu bringen.

Wegen der Vielfalt von Strategien, und weil Global Governance ein politischer Prozeß ist, können die unterschiedlichsten Akteure (aus Politik und Wissenschaft) fast problemlos an das Konzept anknüpfen. Mit dem Konzept der Global Governance wird daher das Terrain für einen Basiskonsens abgesteckt, der es erlaubt, die sozio-ökonomischen Ungleichheiten und ökologischen Krisenszenarien durch die systemische Integration und Kooperation ,alter“ und ,neuer“ Akteure zu lösen. Der Kooperationsansatz soll der Komplexität der Probleme entsprechen, die nicht mehr nur durch die ,invisible hand“ des Marktes und durch die ,visible hand" des Staates bearbeitet werden sollen, sondern in network like forms of governance, also mithilfe der ,third hand“ (Diane Elson) gesellschaftlicher Bewegungen.

Während Politik im Nationalstaat immer und notwendigerweise eine territoriale Dimension hat und Politik Grenzen, gerade territoriale Grenzen setzte, fehlt diese territoriale Dimension im Prozeß der Global Governance. Anstelle der territorialen Verankerung von Politik spielen hier die "Grenzen des Umweltraums" (2) und die Grenzen der sozialen Zumutungen eine beträchtliche Rolle. Sie existieren einerseits ,objektiv“, werden aber im politischen Diskurs von Nicht-Regierungs-Organisationen (engl. Abk: NGOs) und anderen Bewegungen zum Gegenstand der Politik erhoben und von den politischen Institutionen auf allen Ebenen bearbeitet.

\section{NGOS als "nützliche Partner"?}

Allerdings funktioniert die Welt noch nicht nach den Regeln von Global Governance. Denn die westlichen Regierungen diktieren weitgehend die Funktionsweisen und Bedingungen der globalen Deregulation durch Weltbank, Internationalen 
Währungsfonds und die Welthandelsorganisation. Die Institutionen des Marktes meinen, auf demokratische Legitimation gut und gern verzichten $\mathrm{zu}$ können; sie muissen nicht um den sozialen Konsens buhlen. Denn die Legitimation wird auf dem Markt prozedural hergestellt und muß nicht substantiell und politisch hergestellt werden (3). In den ,high politics“, d.h. der Wirtschafts- und Sicherheitspolitik, werden internationale Absprachen nach wie vor hinter verschlossenen Türen getroffen. Ein aktuelles Beispiel sind die Verhandlungen um das Multilaterale Investitionsabkommen (MAI). Die politische Souveränität der nationalen Staaten scheint hier kein Thema zu sein. Die ,neorealistischen“ Theoretiker der internationalen Politik können darauf verweisen, um den Formen der Global Governance tatsächliche Bedeutung abzusprechen.

Das heißt nicht, daß die Weltmarktinstitutionen auf die Kooperation mit NGOs gänzlich verzichten. Ganz im Gegenteil: Sie fragen die Fähigkeiten der NGOs zur Lösung sozialer und ökologischer Weltprobleme partiell und selektiv nach. „Die NRO werden als 'nützliche Partner' in die Global Governance-Architektur inkorporiert, solange sie gebraucht werden, bleiben aber im Konflikt mit mächtigen politischen und wirtschaftlichen Interessen Juniorpartner. Sie bilden also nicht den Organisationskern von Global Governance“ (4). Die globale Menschenrechtspolitik wäre ohne NGOs gar nicht denkbar. Sie werden in der Entwicklungszusammenarbeit, etwa von der Weltbank, als kostengünstige Projektbearbeiter oder Berater und vermeintliche Mittler zwischen Projektträgern und betroffenen Bevölkerungsgruppen berïcksichtigt. Seit den späten 80er Jahren sind auch die umweltpolitischen Organisationen stärker von den großen Organisationen der Weltwirtschaft und -politik gefragt.

Aber nicht nur als Implementationsorgane (vorrangig Entwicklungs-NGOs), als Akteure im Prozeß der Problemidentifikation und -definition (Umwelt-NGOs) oder als Beteiligte an der Entwicklung von Normen (Menschenrechts-NGOs) sind die Selbstorganisationskräfte der Gesellschaft am Werk. Die Partizipation der NGOs am globalen Politikprozeß erbringt ganz offensichtlich auch Legitimationsleistungen. Die supranationalen und globalen Institutionen bedürfen anscheinend trotz der formalen Legitimation durch politische Verfahren und ökonomische Marktprozesse der substantiellen Zuarbeit von NGOs, so wie der demokratische Nationalstaat der Parteien als Mittlerorganisationen zwischen
Gesellschaft und Staat bedurfte (und bedarf). Parteien freilich können etwas, was NGOs nicht vermögen: sie können, wenn sie denn die Mehrheiten gewinnen, die Regierung bilden. Dies können NGOs nicht, weder auf der lokalen noch auf der globalen Ebene. NGOs haben nur bei den „soft issues“, d.h. in den Politikfeldern Ökologie und Soziales, gewisse Informationsrechte und Mitbestimmungsmöglichkeiten (5).

Das läßt sich an den Bedingungen der Teilnahme im UNO-System anschaulich zeigen: Die Regeln der ständigen Konsultation von NGOs basieren im wesentlichen auf der Resolution 1296 aus dem Jahr 1968. Erst als Folge des RioProzesses wurden die Beteiligungsmöglichkeiten Ende 1996 mit der Resolution 1296/31 etwas erweitert. Die rund 1500 NGOs (Stand März 1998) mit Konsultativstatus haben im Wirtschafts- und Sozialrat (ECOSOC) nur sehr eingegrenzte Mitwirkungsmöglichkeiten. Für die Füllung der Strukturen der Global Governance mit demokratischem Leben sicherlich eine höchst unzureichende Bedingung.

\section{Widersprüchliche Rolle}

So ergibt sich aus den unterschiedlichen Funktionen der NGOs in der globalen Politik ein höchst widersprüchliches Resultat, das wenigstens vier zentrale Aspekte hat. NGOs werden erstens im Projekt einer Global Governance aus Gründen der Effizienz und Legitimationsbeschaffung benötigt. Sie sind als (konstruktive) Kritiker, Impulsgeber für alternative Lösungsvorschläge oder Bündnispartner gefragt und erbringen wichtige Transferleistungen. In lokale und globale Netzwerke eingebundene NGOs verknüpfen - flexibler und weitreichender als Parteien oder Gewerkschaften - die verschiedenen (widersprüchlichen) Handlungsräume von der lokalen über die nationale bis zur globalen Ebene. Sie treten als Mediatoren in Erscheinung, artikulieren lokale oder nationale Interessenlagen, engagieren sich in der Menschenrechtspolitik und arbeiten an Umwelt- und Sozialstandards oder der Verrechtlichung der internationalen Klima- und Biodiversitätspolitik mit. Die dabei erarbeiteten Standards und Lösungskonzepte kehren - Bumerangeffekt! in modifizierter Form auf die nationalstaatliche oder kommunale Ebene zurück, wo sie letztlich ihre Wirkung ,entfalten“ müssen.

Aber es existieren zweitens keine Verfahren, die den NGOs verantwortliche Mitentscheidungsrechte übertragen und somit transparente Poli- tikprozesse ermöglichen würden. Offenbar werden die traditionellen "global players“ (Nationalstaaten, supranationale Organisationen, transnationale Konzerne, globale Medienagenturen) ihre Definitions-, Entscheidungs- und Handlungsmacht auch nicht ohne weiteres abtreten, um die Herausbildung einer demokratisch verfaßten Global Governance zu ermöglichen. Darüber hinaus sind die Märkte und die ökonomischen Akteure entscheidend für die Spielräume, die den NGOs in der Sozial- und Umweltpolitik bleiben. NGOs können also Einfluß nehmen; doch letztlich sind politische Macht und Marktökonomie durchsetzungsfähiger als Netzwerke der Zivilgesellschaft.

Die NGO-Community ist drittens aufgrund unterschiedlicher politischer Auffassungen, Werthaltungen, Zielvorstellungen oder Arbeitsweisen äußerst heterogen. Sie umfaßt international wirkende Großorganisationen wie WWF oder Greenpeace, lokale Graswurzel-Organisationen und globale Netzwerke. Völlig unterschiedlich sind oftmals die Ziele, die von NGOs verfolgt werden, und die nicht unbedingt ,zivilen“ Kriterien entsprechen müssen. Auch die Binnenstruktur transnationaler NGONetzwerke ist nicht per se demokratisch verfaßt. Zum einen herrschen hinsichtlich der zur Verfügung stehenden Ressourcen wie Geld und fachliche Kompetenzen große Unterschiede zwischen den NGOs, so daß machtvolle Zentren von ansonsten schwachen Netzwerken entstehen können. Zum anderen definieren sich Netzwerke durch die Zugehörigkeit bestimmter NGOs und haben die Tendenz, sich nach außen zu schließen. Den Netzwerkmitgliedern stehen diejenigen NGOs oder Individuen gegenüber, die aufgrund fehlender fachlicher Kompetenzen, Geldmangel oder fehlender Sprachkenntnisse keinen Zugang zum Netz haben.

Aus dieser Perspektive sind NGOs viertens nicht nur Kritiker und Mahner oder das Korrektiv postfordistischer Regulation, sondern tragen selbst zur Fragmentierung der Weltgesellschaft bei, weil sie nur für eine spezifische Klientel und deren Partikularinteressen die aktive Teilnahme am Prozeß der Globalisierung organisieren (6). Verstärkt wird dies noch dadurch, daß sich Bündnisse und Verhandlungsstrukturen bilden, an denen neben den NGOs Regierungsvertreter und Industrielobbyisten beteiligt sind. Es fehlen nachvollziehbare und öffentliche Entscheidungsprozesse und daher können sich transnationale politische Subsysteme (oder co-governments) institutionalisieren, die sich demokratischen 
Verfahren weitgehend entziehen. Damit steht die zivilgesellschaftliche Legitimation einer Global Governance auf tönernen Füßen.

Nur der Tendenz nach haben sich demokratisch verfaßte überstaatliche Netzwerkstrukturen der globalen Politik gebildet. Es bleibt auch fraglich, ob durch komplizierte globale Verfahren der Partizipation die Herstellung von Kongruenz zwischen den Entscheidungen, Interessen und der Kontrolle, sprich zwischen den Akteuren in den unterschiedlichen politischen Räumen, überhaupt möglich ist. Global governance "Weltordnungspolitik“ - ist heute daher eher Versprechen denn ein Begriff, der tatsächliche Verhältnisse im globalen Politikraum erfaßt.

\section{Anmerkungen}

(1) Stiffung Entwicklung und Frieden ( $\mathrm{Hg}$.): Nachbarn in Einer Welt. Der Bericht der Kommission für Weltordnungspolitik. The Commission on Global Governance, Bonn 1995.

(2) Vgl. BUND, Misereor ( $\mathrm{Hg}$.$) : Zukunftsfähiges Deutsch-$ land. Ein Beitrag zu einer global nachhaltigen Entwicklung, Basel, Boston, Berlin 1996 sowie

Altvater, Elmar, Birgit Mahnkopf: Grenzen der Globalisierung. Ökonomie, Ökologie und Politik in der Weltgesellschaft, Münster 1996, S.503ff.

(3) Altvater/ Mahnkopf, a.a.0. S.503.

(4) Messner, Dirk, Franz Nuscheler (Hg.): Global Governance. Organisationselemente und Säulen einer Weltordnungspolitik, in: Dies.: Weltkonferenzen und Weltberichte. Ein Wegweiser durch die internationale Diskussion, Institut für Entwicklung und Frieden (INEF), Bonn 1995, S.12-36 (hier S.30).

(5) Walk, Heike, Achim Brunnengräber: Die „NGO-Community" im Spannungsfeld von Globalisierungs- und Fragmentierungsprozessen, in: Peripherie, Zeitschrift für Politik und Ökonomie in der Dritten Welt, Schwerpunkt: Globalisierung, Nr. 59/60, 15. Jg., Dezember 1995, S.118-139. (6) Altvater, Elmar, Achim Brunnengräber, Markus Haake, Heike Walk ( $\mathrm{Hg}$.): Vernetzt und verstrickt. Nicht-Regierungs-Organisationen als gesellschaftliche Produktivkraft, Münster 1997.

\section{Die Autorinnen}

Dr. Elmar Altvater ist Professor am Fachbereich Politische Wissenschaft der Freien Universität Berlin. Achim Brunnengräber und Heike Walk sind wissenschaftliche MitarbeiterInnen im von der VolkswagenStiffung geförderten Forschungsprojekt: "Global Governance. NGO-Neizwerke, Nationalstaaten und das internationale Institutionensystem - untersucht am Beispiel der internationalen Klimapolitik" an der FU Berlin. Kontakt: Fachbereich Politische Wissenschaft, Ihnestraße 21, 14195 Berlin, Tel, 030/ 838-4965, E-mail: priklima@zedat.fu-berlin.de

Transnationale Konzerne als (umwelt) politische Akteure der Global Governance

\title{
Brandstifter als Feuerwehr?
}

\author{
Die Global-Governance-Debatte bedeutet eine Horizonterweiterung hin zu \\ einem Einbezug auch nicht-staatlicher Akteure. Erstaunlicherweise wurden \\ jedoch die Transnationalen Konzerne, auf deren enorm gestiegene weltwirt- \\ schaftliche und politische Bedeutung allerorten hingewiesen wird, von bisheri- \\ gen Untersuchungen weitgehend ausgeblendet.
}

G anz gleich, ob dieser Tage ein wichtiges internationales Umweltabkommen verhandelt wird, die Commission on Sustainable Development in New York die weitere Umsetzung der Agenda 21 diskutiert oder die Welthandelsorganisation (WT0) in Genf allmählich auch das Gespräch mit Nichtregierungsorganisationen aufnimmt - immer sind bei solchen Zusammenkünften auch Akteure dabei, deren Rolle in umweltpolitischen Analysen zumeist noch ausgeblendet bleibt: Es sind die Transnationalen Konzerne und ihre Organisationen.

So wie auf nationaler Ebene Unternehmen und ihre Verbände Einfluß auf die Umweltpolitik nehmen, ist auch in der Arena internationaler Umweltpolitik zu beobachten, daß sich Unternehmen und insbesondere Transnationale Konzerne vielfältiger institutioneller Formen und Praktiken bedienen, um Einfluß auf internationale Umweltregime sowie die allgemeine Umweltdebatte $\mathrm{zu}$ gewinnen.

Viele Umweltorganisationen sehen hierin eine große Gefahr und ein Haupthindernis für den Aufbau einer Weltumweltordnung, wie sie als „unverzichtbare Säule“ (1) in der Global Governance-Diskussion anvisiert wird. David Korten kommt in seinem vielbeachteten Buch ,When corporations rule the world" zu der Empfehlung: ,Unternehmen (...) gehören einfach nicht in den politischen Raum der Menschen. (...) Das eigentliche Ziel sollte ein klares Verbot der Beteiligung von profit-orientierten Unternehmen an jeglicher Aktivität sein, die der Beeinflussung des politischen Prozesses dient" (2).

Im Gegensatz zu dieser kritischen Position ist der Bericht der Commission on Global Governance geprägt von einer Rhetorik der ,Nachbarschaft" in der ,einen Welt" und fordert sogar eine noch stärkere Teilnahme der Privatwirtschaft und gerade Transnationaler Konzerne (TNKs) an der globalen Politik. Die Kommission weist zwar auch auf die immer krasser werden- den materiellen, politischen und ökologischen Interessenkollisionen zwischen den Reichen und den Habenichtsen im globalen Kapitalismus hin und macht einige zarte Reformvorschläge. Unstrittig bleibt aber in diesem Diskurs die Rolle der TNKs als legitime internationale Akteure und Beteiligte am globalen Regieren.

Wenn aber die Global Governance-Diskussion mehr als nur normative Visionen globaler Regulierung verbreiten will, sollte sie zunächst analytisch $\mathrm{zu}$ einem angemesseneren Verständnis des tatsächlich stattfindenden „Regierens“ in der Welt beitragen - und in diesem Zusammenhang auch mehr Klarheit über Inhalt und Folgen des Mitregierens von Konzernen in der internationalen Umweltpolitik bringen. Wird hierauf verzichtet, so besteht m.E. Anlaß, der Rhetorik, globaler Nachbarschaft" eine ebenso beißende Kritik an ihren apolitischen Harmonie-Annahmen entgegenzuhalten, wie sie Newton und Harte schon dem Gros der Ökomanagement-Literatur vorgehalten haben (3).

Die hier angedeutete analytische Aufgabe ist bisher noch kaum angegangen worden - und so hat dieser Beitrag sowohl theoretisch als auch empirisch vor allem explorativen Charakter (4). Wenn nun auch Transnationale Konzerne im „Zusammenwirken von staatlichen und nichtstaatlichen Akteuren von der lokalen bis zur globalen Ebene" (5) betrachtet werden sollen, bedarf es eines analytischen Rahmens, der nicht - wie die ökonomische Orthodoxie - die Existenz von Macht ausblendet, aber auch nicht wie viele politikwissenschaftliche Arbeiten - auf die institutionalisierten Machtbeziehungen der Nationalstaaten beschränkt bleibt. Politische Ökonomie tut also not, genauer: Internationale Politische Ökonomie (6).

Die sogenannte neo-gramscianische Schule der Internationalen Politischen Ökonomie (7) erlaubt dabei eine integrierte Analyse erstens grundlegender ökonomisch-materieller Rahmenbedingungen der Umweltpolitik, zweitens 
(c) 20I0 Authors; licensee IÖW and oekom verlag. This is an article distributed under the terms of the Creative Commons Attribution Non-Commercial No Derivates License (http://creativecommons.org/licenses/by-nc-nd/3.o/), which permits unrestricted use, distribution, and reproduction in any medium, provided the original work is properly cited. 